\title{
一类演化方程 $u_{t}=\alpha u^{q} u_{1}+\alpha u_{p}$ 的差分格式
}

\author{
秦 孟 兆 \\ (中国科学院计算中心)
}

文献 [1] 对下列方程

$$
u_{t}=a u^{q} u_{1}+a u_{p}
$$

（其中 $q \geqslant 0, p \geqslant 2, p, q$ 是整数, $\alpha, a$ 是常数, $u_{1}, u_{p}$ 表示 $u$ 对 $x$ 的一次和 $p$ 次导数）的守 恒律的个数有如表 1 的结果.

表 1

\begin{tabular}{|c|c|c|c|c|c|}
\hline$p$ & 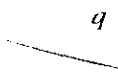 & 0 & 1 & 2 & $\geqslant 3$ \\
\hline 偶 & & 1 & 1 & 1 & 1 \\
\hline \multirow{2}{*}{ 奇 } & 3 & $\infty$ & $\infty$ & $\infty$ & 3 \\
\hline & $>4$ & $\infty$ & 3 & 3 & 3 \\
\hline
\end{tabular}

对这样一类很重要的方程, 如何建立相应的差分格式是一件很有意义的事. 我们知道方程(1) 在某种意义上讲是下列二个方程:

$$
\begin{aligned}
& u_{t}=\alpha u^{q} u_{1}, \\
& u_{t}=a u_{p}
\end{aligned}
$$

的迭加. 文献 [2] 曾对方程 (3) 在 $p=3$ 情况下讨论了各种格式的建立. 本文是将文献 [2] 中结果推广到一般方程 (即 $p$ 是大于零的任何正整数), 分二种情况来讨论, 即 $P$ 是偶数或奇数 二种情况. 得到有趣的结果 (当 $p$ 是奇数的时候) 显式右偏心格式 (这里假设方程 (3) 中系数 $a>0$ ) 仅对 $p=1,5,9,13, \cdots$ 是有条件稳定的. 同样左偏心格式仅对 $p=3,7,11,15 \cdots$ 是有条件稳定的. 当 $a<0$, 情况正好相反, 即右偏心格式仅对 $p=3,7,11,15 \cdots$ 是有条件 稳定的, 而左偏心格式仅对 $p=1,5,9,13, \cdots$ 是有条件稳定的. 更有意思的是这类偏心格 式与微分方程的边界条件是配匹的. 例如当 $a>0, p=3$. 为使差分格式能向前推进, 左边 必须给二个边界条件, 右边给出一个边界条件. 而微分方程也正是这样给边界条件才是适定 的. 对隐式偏心格式也有类似的情况. 最后讨论对方程 (1) 如何建立相应的差分格式.

方程 (3) 当 $p$ 是偶数时, 我们把它写成

当 $p$ 是奇数时,我们把它写成

$$
u_{t}=\left(a \cdot u_{p-2}\right)_{x x} .
$$

$$
u_{t}=\left(a u_{p-1}\right)_{x} .
$$

显然方程 (3) 当 $p$ 是奇数时, 初值问题是适定的. 同样当 $p$ 是偶数时为使方程 (3) 适定, 本文 1980 年 3 月 31 日收到.

第 5 期 
我们取 $a=(-1)^{\frac{p}{2}-1}$. 方程 (3) 归根到底是下列这些格式(表 2).

表 2

(1)
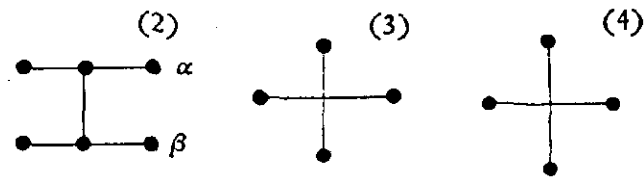

(4)

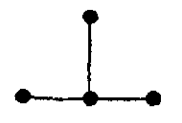

(2)

(3)

)
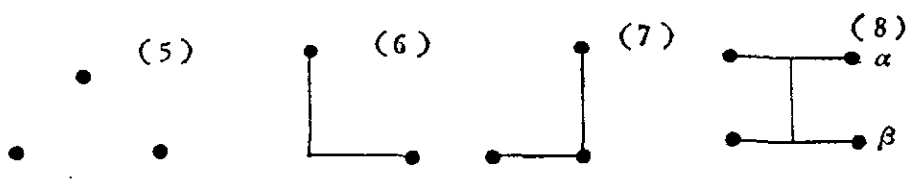

(9)

(10)

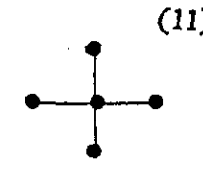

(11)

(12)

$\left(12^{\prime}\right)$
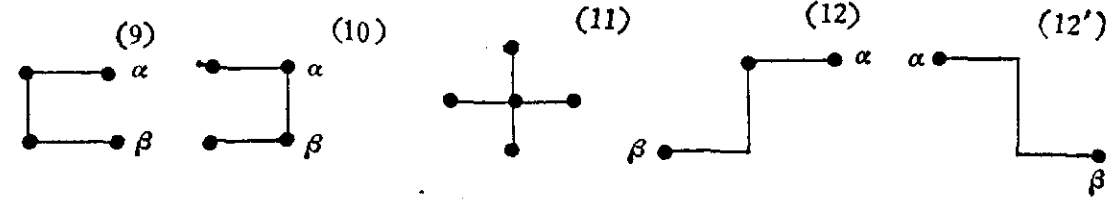

命题 1 方程 $u_{t}=(-1)^{\frac{p}{2}-1} u_{p}$, 其中 $p$ 是偶数, 则

（i）格式 (1) 的稳定性条件为 $\frac{\Delta t}{\Delta x^{p}} \leqslant \frac{1}{2^{p-1}}$;

（ii）格式 (2) 当 $\alpha \geqslant \frac{1}{2}, \alpha+\beta=1$ 是绝对稳定的;

(iii) 格式 (3) 是绝对不稳定的;

（iv）当 $p>2$, 显式 Dufort-Frankel 格式是绝对不稳定的, 当 $p=2$, 或隐式 Dufort-Frankel 格式是绝对稳定的.

命题 2 对方程 $u_{t}=a u_{p}$ (其中 $p$ 是奇数).

(i) 格式 (5) 是绝对不稳定的;

（ii）格式 (6) 的稳定性条件为 $(-1)^{\frac{p-1}{2} a}>0,|a| \frac{\Delta t}{\Delta x^{p}} \leqslant \frac{1}{2^{p-1}}$;

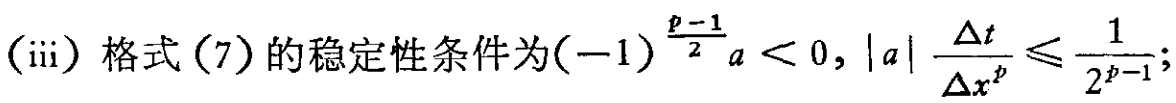

(iv) 对称 Crank-Nickolson 格式(简称 $\mathrm{C}-\mathrm{N}$ ) (8) 是绝对稳定的, 当 $\alpha \geqslant 1 / 2, \alpha+\beta=1$;

（v）右偏 $\mathrm{C}-\mathrm{N}$ 格式(9)是绝对稳定的, 当 $(-1)^{\frac{p-1}{2}} a>0, \alpha \geqslant 1 / 2$;

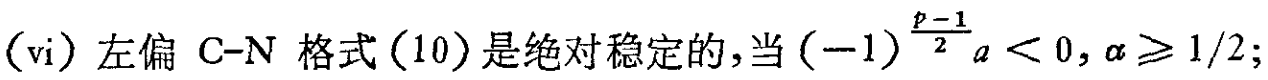

（vii）青蛙跳格式 (11) 的稳定性条件是 $|a| \frac{\Delta t}{\Delta x^{p}} \leqslant \frac{(1+p)(\sqrt{1+p})^{p-1}}{2^{p}(\sqrt{p})^{p}}$;

（viii）反 Z字型格式 (12):

1) 当 $(-1)^{\frac{p-1}{2}} a>0$, 格式 (12) 绝对稳定性条件是 $\alpha \geqslant 1 / 2$, 而当 $(-1)^{\frac{p-1}{2}} a<0$, 稳定 性条件为

$$
|a| \frac{\Delta t}{\Delta x^{p}} \leqslant \frac{1}{2^{p-1}(1-2 \alpha)}, \quad \alpha \leqslant 1 / 2
$$

正 Z 字型格式 (12)'。 
2) 当 $(-1)^{\frac{p-1}{2}} a<0$, 格式 $\left(12^{\prime}\right)$ 绝对稳定性条件是 $a \geqslant 1 / 2$, 而当 $(-1)^{\frac{p-1}{2}} a>0$, 稳 定性条件为

$$
|a| \frac{\Delta t}{\Delta x^{p}} \leqslant \frac{1}{2^{p-1}(1-2 \alpha)}, \quad \alpha \leqslant 1 / 2 .
$$

命题 3 对方程 $u_{t}=u^{q} u_{1}+u_{p}$,

其中 $p$ 是奇数, Kruskal-Zabusky 格式线性稳定性条件为

$$
\frac{\Delta t}{\Delta x^{p}} \leqslant \frac{1}{\Delta x^{p-1}\left|u_{0}^{q}\right|+2^{\frac{p+1}{2}}} .
$$

\section{注记}

(i) 当 $p=2$, 命题 1 的结果与一般热传导方程各类格式结果一致 ${ }^{[3]}$.

(ii) 当 $p=1,3$, 命题 2 的结果与单向波中各类格式 ${ }^{[4]}$ 和色散方程中各类结果一致 ${ }^{[2]}$.

(iii) 当 $q=1, p=3$, 命题 3 的结果与文献 [5] 中分析 Kdv 方程 Kruskal-Zabusky 格式 结果一致。

致谢：本文承冯康教授热情支持与鼓励,作者表示衷心的感谢.

\section{参考文献}

[1] 屠规彰、秦孟兆,中国科学, 1980, 5: 421-432.

[2] 秦孟兆,方程 $u_{t}=a u_{x \times x}$ 的差分格式,计算数学,即将发表.

[ 3 ] Richtmyer, R. D. \& Morton, K. W., Difference Methods for Initial-value Problems, New York, 1967.

[4] 冯康,数值计算方法,国防工业出版社,北京, 1978 .

[5] Chris Eilbeck, J., Numerical Studies of Solitons, Oxford, 1978. 